\title{
The Importance of Assessing Burning and Stinging when Managing Rosacea: A Review
}

\author{
Martin SCHALLER ${ }^{1}$, Thomas DIRSCHKA², Sol-Britt LONNE-RAHM³ ${ }^{3}$, Giuseppe MICALI ${ }^{4}$, Linda STEIN-GOLD ${ }^{5}$, Jerry TAN ${ }^{6}$ and \\ James DEL ROSSO ${ }^{7}$ \\ ${ }^{1}$ Department of Dermatology, Universitatsklinikum Tuebingen, Tuebingen, Baden-Württemberg, ${ }^{2}$ CentroDerm-Clinic, Wuppertal and Faculty \\ of Health, University of Witten-Herdecke, Witten, Germany, ${ }^{3}$ Department of Medicine Solna, Dermatology and Venereology Unit, Karolinska \\ Institutet, Stockholm, Sweden, ${ }^{4}$ Dermatology Clinic, University of Catania, Catania, Italy, ${ }^{5}$ Department of Dermatology, Henry Ford Health \\ System, West Bloomfield, MI, USA, ${ }^{6}$ Windsor Clinical Research Inc. and Department of Medicine, University of Western Ontario, Windsor, \\ ON, Canada and 'JDR Dermatology Research and Thomas Dermatology, Las Vegas, NV, USA
}

\begin{abstract}
Rosacea, a chronic condition usually recognized by its visible presentation, can be accompanied by invisible symptoms, such as burning and stinging. The aim of this review is to gather the most recent evidence on burning and stinging, in order to further emphasize the need to address these symptoms. Inflammatory pathways can explain both the signs and symptoms of rosacea, but available treatments are still evaluated primarily on their ability to treat visible signs. Recent evidence also highlights the adverse impact of symptoms, particularly burning and stinging, on quality of life. Despite an increasing understanding of symptoms and their impact, the management of burning and stinging as part of rosacea treatment has not been widely investigated. Clinicians often underestimate the impact of these symptoms and do not routinely include them as part of management. Available therapies for rosacea have the potential to treat beyond signs, and improve burning and stinging symptoms in parallel. Further investigation is needed to better understand these benefits and to optimize the management of rosacea.
\end{abstract}

Key words: rosacea; stinging; burning; management; symptom; invisible.

Accepted Oct 13, 2021; Epub ahead of print Oct 13, 2021

Acta Derm Venereol 2021; 101: adv00584.

Corr: Martin Schaller, Department of Dermatology, Universitatsklinikum Tuebingen, Tuebingen, Baden-Württemberg, Germany. E-mail: Martin. Schaller@med.uni-tuebingen.de

$\mathrm{R}$ osacea is a chronic inflammatory skin disease predominantly affecting the central face, which is most often diagnosed through assessment of visible signs alone $(1,2)$. These include erythema, inflammatory lesions, phymatous changes and/or ocular manifestations $(1,3)$. The disease is also characterized by an array of invisible symptoms, of which burning and stinging sensations are common (4). Other invisible symptoms of rosacea, such as itching, are beyond the scope of this review. Burning in rosacea is defined as "an uncomfortable or painful feeling of heat, typically in the centro-facial region" (1). Stinging in rosacea is defined as "an uncomfortable or painful sharp, pricking sensation, typically in the centrofacial region" (1).

\section{SIGNIFICANCE}

Rosacea, which is usually recognized by its visible presentation, can be accompanied by symptoms such as burning and stinging, which are not new features of this chronic disease. By gathering the most recent evidence on burning and stinging in rosacea, it is hoped that clinicians will begin to consciously consider the impact of these symptoms on patients' quality of life and start to include them as part of their rosacea assessment and disease management approach. Future clinical studies will provide further evidence on the ability of available therapies for rosacea to improve burning and stinging symptoms in parallel with visible signs.

A global survey performed across 6 countries (Canada, France, Germany, Italy, Poland and the USA), which included patients with rosacea $(n=300)$ and patients with psoriasis with facial involvement $(n=318)$ revealed that more than $60 \%$ of patients surveyed regardless of disease were mostly bothered by the physical discomfort of their disease. The symptoms assessed in the survey included burning, stinging, itching and pain (2). Another global survey $(\mathrm{N}=710)$ which evaluated the impact of rosacea signs and symptoms on overall disease burden found that symptoms were significantly associated with a higher disease burden. These high-burden patients also spent significantly more time, on average, on daily skincare because of their rosacea $(p<0.01)(5)$. Collectively, this evidence demonstrates the level of impact that symptoms such as burning and stinging can have on patients with rosacea.

Furthermore, most clinical practice guidelines on rosacea recommend that the primary treatment objective is clearing the visible signs of the disease; the invisible symptoms are not always addressed $(6,7)$. The "Beyond the Visible 2018" report investigating the burden faced by patients with rosacea illustrated the disconnect between physicians and patients - physicians often overestimate the visible signs of rosacea, while underestimating and overlooking the symptom experience for patients (6).

Given the multiple signs and symptoms experienced by patients with rosacea, there is a need to address symptoms that are bothersome to patients but not always addressed. There is also a need to alleviate these symptoms 
alongside the visible signs of the disease. All treatments currently available for rosacea are indicated to treat the visible signs of the disease; however, comprehensive management of the disease, including both signs and symptoms, may serve to further improve therapeutic outcomes and the quality of patients' lives $(4,6,7)$.

For this review, published literature on burning and/or stinging in patients with any type of rosacea over the past 5 years were searched and reviewed with the following criteria on PubMed: ( $i$ ) rosacea AND burning in any field (79 results); (ii) rosacea AND stinging in any field (43 results); (iii) rosacea AND burning OR stinging in any field ( 88 results); (iv) rosacea AND sensitive in any field (363 results); and (v) rosacea AND sensitive AND burning OR stinging in any field (14 results).

This review aims to address the available evidence on burning and stinging in patients with rosacea and explores the need to address these symptoms in the treatment and management of rosacea, in order to improve patient outcomes.

\section{BURNING AND STINGING ARE NOT NEW FEATURES}

In 2002, the National Rosacea Society (NRS) in the USA categorized rosacea into 4 major subtypes: erythematotelangiectatic (ETR), papulopustular (PPR), phymatous (PHY) and ocular (OR) (8). This subtype-based classification formed the foundation of subsequent evidence generation and publications in rosacea. Although this classification is focused on the visible presentation of the disease, primarily for greater diagnostic accuracy, it does not emphasize the clinical relevance of associated symptoms, such as burning and stinging, experienced by the patient (8). Evidence shows burning and stinging commonly reported by patients with rosacea may be experienced across all subtypes of rosacea.

- ETR is characterized by persistent centro-facial erythema, flushing and telangiectasia (8). In a 2017 study, $13.9 \%$ of patients with ETR $(n=409)$ reported burning or stinging as bothersome symptoms of their disease $(p<0.01$ vs PPR; $24.1 \%, n=191)(9)$.

- The PPR subtype is characterized by persistent centrofacial erythema with transient papules or pustules in a central facial distribution (8). In a study exploring the benefit of combination treatment for subjects with severe PPR $(\mathrm{N}=273)$, at least $85 \%$ of subjects experienced burning and stinging at baseline. In this study, rosacea severity was measured by the Investigator's Global Assessment (IGA) score, which is a 5-point scale in which IGA 0 is "clear" of rosacea lesions or erythema and IGA 4 is indicative of severe rosacea (4).

- The PHY subtype rosacea includes thickening skin, irregular surface nodularities, and enlargement, which occurs most commonly as rhinophyma, especially in the nasal area (8). There are limited data available on burning and stinging in PHY; however, according to the NRS, this subtype is frequently observed in combination with ETR and PPR, hence burning and stinging symptoms may also be present, although further investigation is needed (8).

- OR is characterized by a foreign body sensation in the eye, burning or stinging, dryness, itching, ocular photosensitivity, blurred vision, telangiectasia of the sclera or other parts of the eye, or periorbital oedema (8). A global survey $(\mathrm{N}=300)$ found that OR rosacea patients $(20 \%$ of rosacea patients surveyed) reported burning (53\%) and stinging (38\%) as 2 common skin symptoms (2).

Burning or stinging was also common in neurogenic rosacea, an additional rosacea subtype proposed in a 2011 study. Patients in this group experienced prominent neurological symptoms, of which burning or stinging was the most common symptom, experienced by $100 \%$ of patients $(\mathrm{N}=14)$ alongside erythema (10). Although a small sample size, these findings warrant further investigation.

With advances in available evidence and a need to take on a more patient-centric approach to rosacea treatment, there was a need to progress beyond the subtype classification. A new approach would help to better encompass all signs and symptoms experienced by rosacea patients. In 2013, the American Acne and Rosacea Society (AARS) proposed a phenotype approach to better diagnose and treat patients with rosacea for all signs and symptoms experienced (11). Subsequently, the global ROSacea COnsensus (ROSCO) panel in 2017 supported the transition to a phenotype approach to provide a basis for local guideline development and to help to improve outcomes in all patients with rosacea by individualizing management (12). In 2019, the panel incorporated burning and stinging as secondary diagnostic criteria of rosacea, to be considered as part of any severity assessment (1).

Although the presence of burning and stinging across rosacea subtypes is clear, clinical evidence on these symptoms with proven treatment and management guidance are not readily available. Given this lack of guidance, the need to include these symptoms as a part of diagnosis and management of rosacea may often be overlooked in clinical practice $(2,8,11)$.

\section{Role of neurovascular dysregulation}

In 1999, researchers were already discussing the pathophysiology of stinging sensations in patients with rosacea and a potential link with sensitive skin (13). Neurovascular dysregulation and an altered immune response are both thought to be key pathophysiological elements involved in the burning and stinging response (14). Patients with rosacea are believed to have an increased density of 

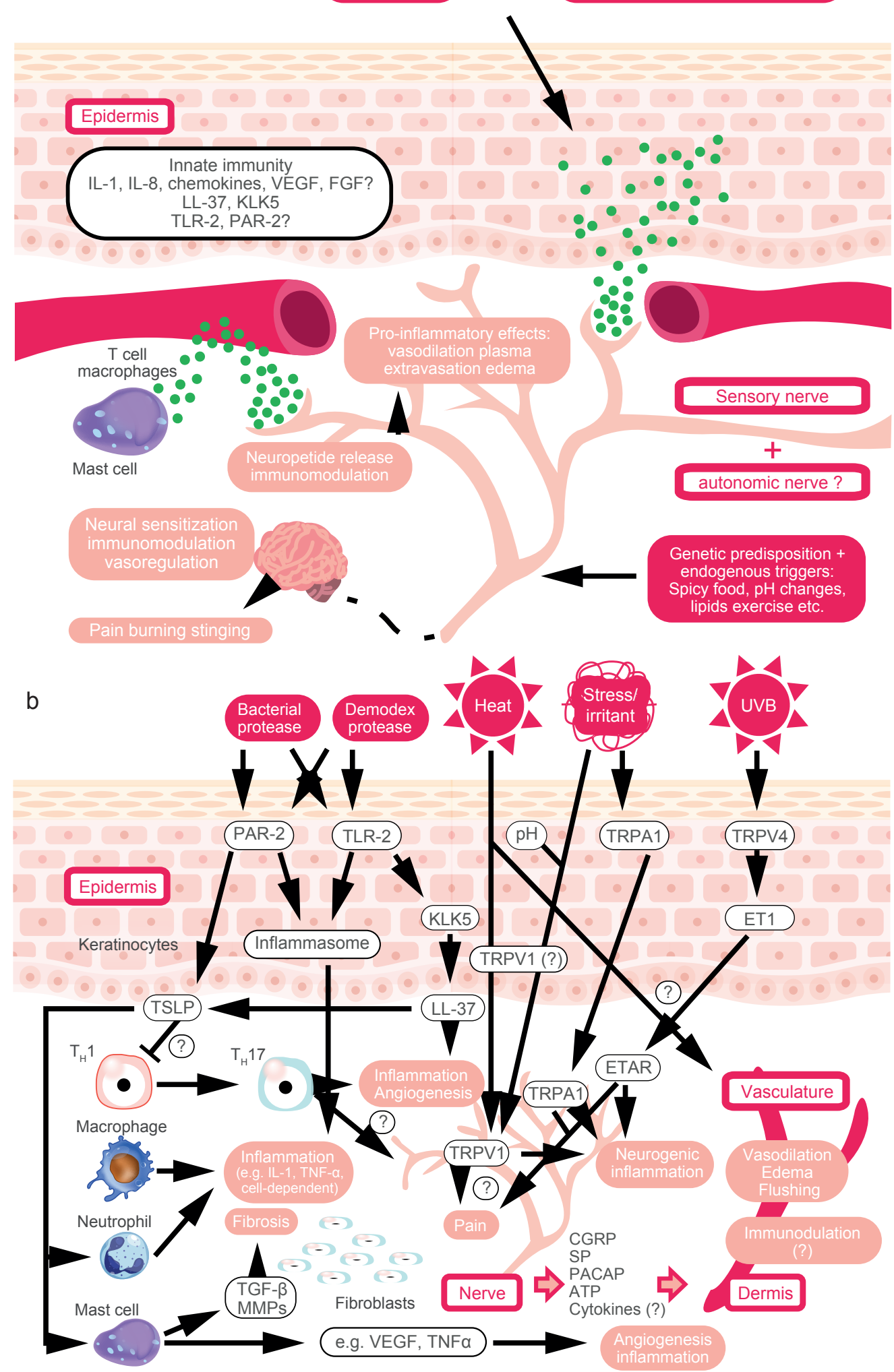

Fig. 1. (a) Previous vs (b) current understanding of rosacea pathophysiology and the believed role of neurovascular transient receptor potential ion channels of vanilloid types (TRPVs) in disease pathogenesis. (a) Adapted from: Steinhoff et al. (16), (b) Buddenkotte and Steinhoff (17). ATP: adenosine triphosphate; CGRP: calcitonin gene-related peptide; ET: endothelin; ETAR: endothelin A receptor; FGF: fibroblast growth factor; IL: interleukin; KLK: kallikrein; LL-37: cathelicidin peptide; MMP: matrix metalloproteinase; PACAP: pituitary adenylate cyclase-activating peptide; PAR: protease activated receptor; pH: potential of hydrogen; SP: substance P; TGF- $\beta$ : transforming growth factor-beta; $\mathrm{T}_{H}$ : T-helper cell; TLR: toll-like receptor; TNF-a: tumour necrosis factor-alpha; TRPA: transient receptor potential ion channels of ankyrin type; TSLP: thymic stromal lymphopoietin; UVB: ultraviolet B-rays; VEGF: vascular endothelial growth factor. Permissions have been obtained from authors and journals for the use of these images. 
transient receptor potential ion channels of vanilloid type (TRPV) on sensory neurones, vascular cells and immune cells. TRPV activation can lead to transient experiences of flushing and burning pain as a result of the release of vasoactive neuropeptides $(15,16)$. In patients with rosacea, TRPV levels can be elevated and eventually become hyperactive, impacting local immune function, vascular regulation, nociception, and epidermal barrier integrity (15). TRPV hyperactivity can also result in sustained flushing (a feeling of warmth), neurogenic inflammation (with oedema) and inflammatory cell infiltration, which can be extensive (Fig. 1) (16, 17). Dysregulation of TRPVs may play a critical role in the mediation of pathophysiological cascades in rosacea and associated symptoms, such as inflammation, flushing, hypersensitive skin, burning and stinging. These findings suggest that the TRPV ion channels may be a possible target for the treatment of both signs and symptoms of rosacea $(15,16)$.

\section{Impact of Demodex mite proliferation}

In 1993, studies using the standardized skin surface biopsy (SSSB) approach observed that Demodex mite density was between 3.5 times and 18 times higher in subjects with PPR than in healthy controls $(18,19)$. A comparison between one of these studies and a 2005 study using SSSB demonstrated Demodex mite density was as much as 51 times higher in subjects with PPR than in healthy controls (Fig. 2) (18, 20, 21). The presence of higher Demodex mite densities in rosacea compared with control skin has also been confirmed by other authors and using different techniques, including a 2012 PCR study that found density was 5.7 times and 2.9 times higher in subjects with rosacea with ETR/facial PPR or facial PPR than in healthy controls, respectively (22). The causal relationship between Demodex mites and rosacea is based not only on their higher density in patients with rosacea compared with healthy controls; other factors include histological damage and perifollicular infiltrate caused by the mite, possible activation of the toll-like receptor 2

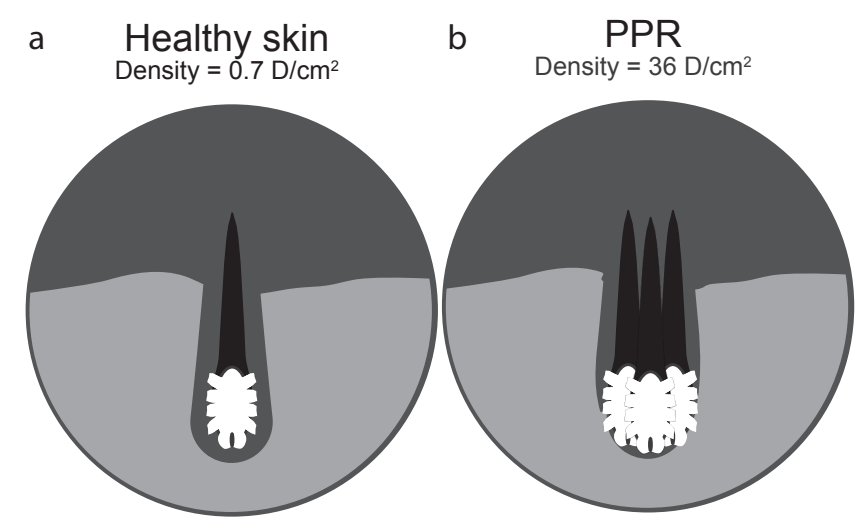

Fig. 2. Cutaneous Demodex mite density in (a) healthy skin vs (b) papulopustular rosacea (PPR) using the standardized skin surface biopsy approach. Adapted from: Forton et al. (18, 20, 21). D: Demodex.
(TLR-2) pathway leading to inflammatory skin changes, clinical improvement with pure acaricidal treatments and other arguments explained below $(21,23)$.

There is evidence that proliferation of Demodex mites in facial skin can contribute to the emergence of clinical manifestations of rosacea $(21,24,25)$. In a prospective cohort study, the authors showed a highly significant reduction in Demodex mites within the first week of topical ivermectin treatment, but also a significant down-regulation of the pro-inflammatory genes interleukin-8 (IL-8), cathelicidin peptide LL-37, beta-defensin-3 (HBD3), TLR-4 and tumour necrosis factor-alpha (TNF- $\alpha$ ), concomitantly with significant clinical improvement of both signs and symptoms of rosacea (24).

Proliferation of Demodex mites causes cutaneous barrier damage by eroding the epithelium. This, in turn, can lead to skin hypersensitivity, which can be reversible when the Demodex mite density is reduced through treatment (21). The underlying pathogenesis is not fully understood; however, there is speculation that chitin, which constitutes part of the Demodex mite's outer shell, plays a role in inducing inflammatory reactions in rosacea (26). TLRs act as sensors of microbial pathogens and trigger downstream immune responses, in order to eliminate the pathogen (15). Chitin induces the expression of TLR-4, suggesting that chitin is sensed by TLRs on keratinocytes and thus could play a role in how Demodex mite infiltration contributes to cutaneous inflammation observed in rosacea (26).

There is evidence to support that individuals with ETR have higher mean densities of Demodex mites than healthy controls, but lower densities than patients with PPR (27). The association between Demodex mites and cutaneous inflammation is controversial, as the skin of individuals without rosacea may also be colonized by Demodex mites as a commensal organism (28). As a result, it is difficult to confirm that high Demodex mite density is a primary cause of burning and stinging in patients with rosacea; however, the stimulation of the immune response by a higher Demodex mite density may play a contributory role (26-30).

Proliferation of Demodex mites is reported to play an important role in the pathophysiology of OR. An inflammatory immune response and an increased density of Demodex mites causes substantial upregulation in proinflammatory cytokines involved in ocular inflammation and vasoregulation, along with enlarged, dilated vessels in the upper dermis. This promotes leukocyte infiltration and post-capillary oedema, which can manifest as blepharitis (31).

\section{Epidermal barrier damage}

Evidence also shows that patients with PPR experience impaired epidermal barrier function. Despite recent developments in the role of Demodex mite colonization 
and neurovascular dysregulation in rosacea, there is still a limited understanding of the correlation between the clinical manifestations of rosacea and dysfunction of the epidermal barrier (32).

Epidermal barrier dysfunction can reduce skin resistance to irritants and allergens, which can exacerbate signs and symptoms of rosacea (33-36). Although the mechanisms are not fully understood, evidence suggests that the interactions between rosacea-prone skin and physical, chemical and microbial factors play important roles in the pathophysiology of rosacea $(15,37)$. PPR is often misdiagnosed as acne vulgaris. In a study comparing the 2 conditions, significantly more subjects with PPR $(n=463)$ presented with erythema, burning, dryness and itching vs acne vulgaris subjects $(n=412)(p<0.05)$ (33). Although data are limited, the consequences of epidermal barrier dysfunction appear to contribute to the pathophysiology, signs, and symptoms of rosacea.

Transepidermal water loss (TEWL) has been shown to be significantly higher in PPR sites vs healthy subjects $(p<0.001)$ (33). A 2020 study found that the epidermis of patients with PPR $(n=25)$ had a lower water content and higher TEWL in lesions than in control areas (38). Mild cleansers and moisturizers have been found to relieve burning and stinging symptoms and improve epidermal barrier function by increasing skin water content and decreasing TEWL $(34,39,40)$. Improvement in epidermal barrier function can reduce signs and symptoms of rosacea by mitigating inflammation and potentially decreasing adverse interactions with cutaneous irritants $(30,32)$.

\section{BURNING AND STINGING ARE AN IMPORTANT PART OF DIAGNOSIS}

As previously discussed, a phenotype approach to diagnosis of rosacea has been strongly proposed, along with a patient-centric approach to disease management by
Table I. Summary of recommendations for rosacea diagnosis according to the 2002 National Rosacea Society (NRS) subtype approach and 2017 global ROSacea COnsensus panel (ROSCO)/ NRS phenotype approaches. (Adapted from: Tan et al. (41))

\begin{tabular}{|c|c|}
\hline \multicolumn{2}{|l|}{2002 NRS (8) } \\
\hline Primary (diagnostic) features ${ }^{a}$ & $\begin{array}{l}\text { Flushing/transient erythema } \\
\text { Non-transient/persistent erythema } \\
\text { Papules and pustules } \\
\text { Telangiectasia }\end{array}$ \\
\hline Secondary features ${ }^{b}$ & $\begin{array}{l}\text { Burning/stinging sensation } \\
\text { Oedema } \\
\text { Dry appearance } \\
\text { Ocular manifestations } \\
\text { Plaque } \\
\text { Peripheral location } \\
\text { Phymatous changes }\end{array}$ \\
\hline \multicolumn{2}{|l|}{2017 ROSCO/2017 NRS $(12,42)$} \\
\hline Diagnostic features $^{c}$ & $\begin{array}{l}\text { Persistent centro-facial erythema with periodic } \\
\text { intensification by potential trigger factors } \\
\text { Phymatous changes }\end{array}$ \\
\hline Major features $^{d}$ & $\begin{array}{l}\text { Transient centro-facial erythema } \\
\text { Inflammatory papules/pustules } \\
\text { Telangiectasia } \\
\text { Ocular manifestations }\end{array}$ \\
\hline Minor/secondary features ${ }^{b}$ & $\begin{array}{l}\text { Burning/stinging sensation } \\
\text { Oedema } \\
\text { Dry sensation/appearance }\end{array}$ \\
\hline
\end{tabular}

According to the 2002 subtyping system, multiple features were grouped into defined subtypes. The 2017 phenotyping system enables various combinations of diagnostic, major and minor features into an individual phenotype.

${ }^{a}$ The presence of one or more primary (diagnostic) feature with a centro-facial

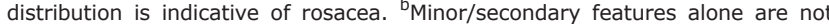

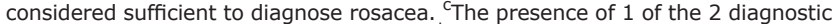
features is sufficient to diagnose rosacea. ${ }^{d}$ In the absence of a diagnostic feature, a combination of at least 2 of the major features is sufficient to diagnose rosacea. Individual publications also discuss the importance of proper history taking, exclusion criteria and trigger factors for specific features.

shifting the focus to presenting signs and symptoms (Table I) $(8,12,41,42)$. There has been a growing acceptance of this approach in diagnosing and managing rosacea, but many clinicians and authors still depend on subtyping when communicating about rosacea $(1,30$, 41, 43, 44).

Although diagnostic features are mainly visible signs, the 2019 ROSCO panel highlighted that non-visible cutaneous features, which are the symptoms of rosacea, need to be considered as part of any severity assessment, of which burning and stinging are addressed (Table II). It is also important to note that the 2019 ROSCO consensus recommendations included burning and stinging

Table II. Diagnostic features of rosacea as defined by the 2019 global ROSacea COnsensus panel. (Adapted from: Schaller et al. (1))

\begin{tabular}{|c|c|c|}
\hline $\begin{array}{l}\text { Diagnostic } \\
(\geq 1 \text { feature is diagnostic) }(12)\end{array}$ & $\begin{array}{l}\text { Major } \\
(\geq 2 \text { features are diagnostic) (12) }\end{array}$ & Secondary \\
\hline Persistent erythema & Inflammatory papules & Burning sensation \\
\hline $\begin{array}{l}\text { Fixed centro-facial redness in a characteristic pattern that } \\
\text { may periodically intensify in response to triggers. This may } \\
\text { be difficult to assess in darker skin phototypes ( } \mathrm{V} \text { and } \mathrm{VI})\end{array}$ & and pustules & $\begin{array}{l}\text { Uncomfortable feeling of heat } \\
\text { Duration, frequency, intensity, extent (areas involved), } \\
\text { associations with flushing, triggers, impact on daily life }\end{array}$ \\
\hline Phymatous changes & Flushing/transient & Stinging sensation \\
\hline \multirow[t]{4}{*}{$\begin{array}{l}\text { Facial skin thickening due to fibrosis and/or sebaceous } \\
\text { glandular hyperplasia. Most commonly affecting the nose } \\
\text { where it can cause a bulbous appearance }\end{array}$} & $\begin{array}{l}\text { centro-facial erythema } \\
\text { Temporary increase in centro-facial } \\
\text { redness. Can include warm, heat, burning } \\
\text { and/or pain sensations }\end{array}$ & $\begin{array}{l}\text { Uncomfortable or painful sharp, pricking sensation } \\
\text { Duration, frequency, intensity, extent (areas involved), triggers, } \\
\text { characteristic of the sensation, impact on daily life }\end{array}$ \\
\hline & Telangiectasia & Oedema \\
\hline & $\begin{array}{l}\text { Visible vessels in the centro-facial region } \\
\text { extending beyond the } \\
\text { alar area }\end{array}$ & $\begin{array}{l}\text { Facial swelling. Can be soft or firm (non-pitting) and may be } \\
\text { self-limited in duration or persistence } \\
\text { Duration, frequency, degree of swelling (depth, pitting and } \\
\text { distortion), extent (areas involved), daily fluctuation, impact } \\
\text { on daily life }\end{array}$ \\
\hline & Ocular manifestations (12) & $\begin{array}{l}\text { Dryness } \\
\text { Feeling of roughened skin. May be tight, scaly and/or itchy } \\
\text { Duration, frequency, intensity, extent (areas involved), pruritus, } \\
\text { roughness, scale, tightness, peeling, how often moisturizers } \\
\text { needed to be applied, impact on daily life }\end{array}$ \\
\hline
\end{tabular}


as non-visible symptoms that can commonly co-exist with other features of rosacea (1).

The 2019 ROSCO update includes additional considerations to be made by assessing physicians when reviewing the signs and symptoms of rosacea to better treat patients with rosacea (Table II) (1). Adopting such an approach could improve patient outcomes by addressing an individual patient's current clinical presentation and additional concerns (i.e. effects on quality of life) (12).

Despite the acknowledgement of burning and stinging through the phenotype approach in diagnosis and management, a practical tool to specifically score burning and stinging related to rosacea currently does not exist and highlights an opportunity for practical improvements. In addition, many clinicians have not fully incorporated the impact of non-visible symptoms to adequately recognize and address them when treating patients with rosacea (Fig. 3) (6).

\section{BURNING AND STINGING ARE OFTEN NOT RECOGNIZED AND ADDRESSED}

Clinicians often do not recognize or incorporate the impact of symptoms of rosacea. They will, however, strongly emphasize the visible signs of the disease, as compared with patients' perceptions about their rosacea (Fig. 3) (6).

A recent survey (2020) highlighted that when doctors $(\mathrm{N}=361)$ were asked what they typically investigated in their patients with rosacea and psoriasis seen in consultation for the first time, only a minority cited symptoms. Doctors were approximately $30 \%$ more likely to investigate non-visible symptoms in their new patients with psoriasis vs those with rosacea (absolute reduction: $13 \%, 40 \%$ psoriasis vs $27 \%$ rosacea; $p<0.01$ ). When physicians were also asked what they typically investigated in their new and follow-up patients with rosacea as an open-ended question, there were very few mentions of burning and stinging (Table III) (2).
Table III. Invisible symptoms reported by patients with rosacea with moderate disease impact vs invisible symptoms investigated by dermatologists/physicians. (Adapted from: Steinhoff et al. (2))

\begin{tabular}{|c|c|c|c|c|}
\hline & \multicolumn{2}{|c|}{ Rosacea patients $(n=300)$} & \multicolumn{2}{|c|}{$\begin{array}{l}\text { Doctor-investigated } \\
\text { symptoms }(n=361)\end{array}$} \\
\hline & $\begin{array}{l}\text { Patient } \\
\text { experienced } \\
\text { ever }\end{array}$ & $\begin{array}{l}\text { Average impact } \\
\text { on } Q \text { oL out of } \\
10(n)^{\mathrm{a}}\end{array}$ & $\begin{array}{l}\text { New } \\
\text { patients }\end{array}$ & $\begin{array}{l}\text { Follow-up } \\
\text { patients }\end{array}$ \\
\hline Burning & $44 \%$ & $6.7(129)$ & $16 \%$ & $13 \%$ \\
\hline Stinging & $29 \%$ & $6.6(88)$ & $2 \%$ & $1 \%$ \\
\hline Itching & $55 \%$ & $6.5(162)$ & $10 \%$ & $8 \%$ \\
\hline Pain/soreness ${ }^{b}$ & $21 \%$ & $6.7(60)$ & $5 \%$ & $3 \%$ \\
\hline
\end{tabular}

${ }^{a}$ Quality of life (QoL) impact reported by patients was measured on a scale from 0 to 10 , where 0 meant the symptom had no impact on their QoL, while a score of 10 meant the symptom extremely impacted their QoL. 'boreness at affected areas, around patches.

These data demonstrate the need to consistently include symptoms such as burning and stinging during assessment of patients presenting with rosacea. Furthermore, they highlight the need to address these symptoms when designing the management plan.

\section{MANAGEMENT NEEDS TO ENCOMPASS ASSESSMENT OF SYMPTOMS OF ROSACEA}

Most available guidelines and consensus recommendations in rosacea focus on treating visible signs of the disease (Table IV) $(1,45-47)$. The aim now is to improve our approach to rosacea management even further by consistently assessing rosacea symptoms, especially burning and stinging. It is important to recognize that reducing the impact of burning and stinging may also improve patient QoL $(4,48)$.

Given the adverse physical and psychosocial factors experienced by patients with rosacea, there is a need to address this burden beyond just alleviating the visible signs of the disease by aiming for "clear" (IGA 0) (6). Although this treatment approach focuses on visible improvement, the benefits of reaching "clear" (IGA 0) can extend beyond the visible benefits including improvement in quality of life $(1,49)$.

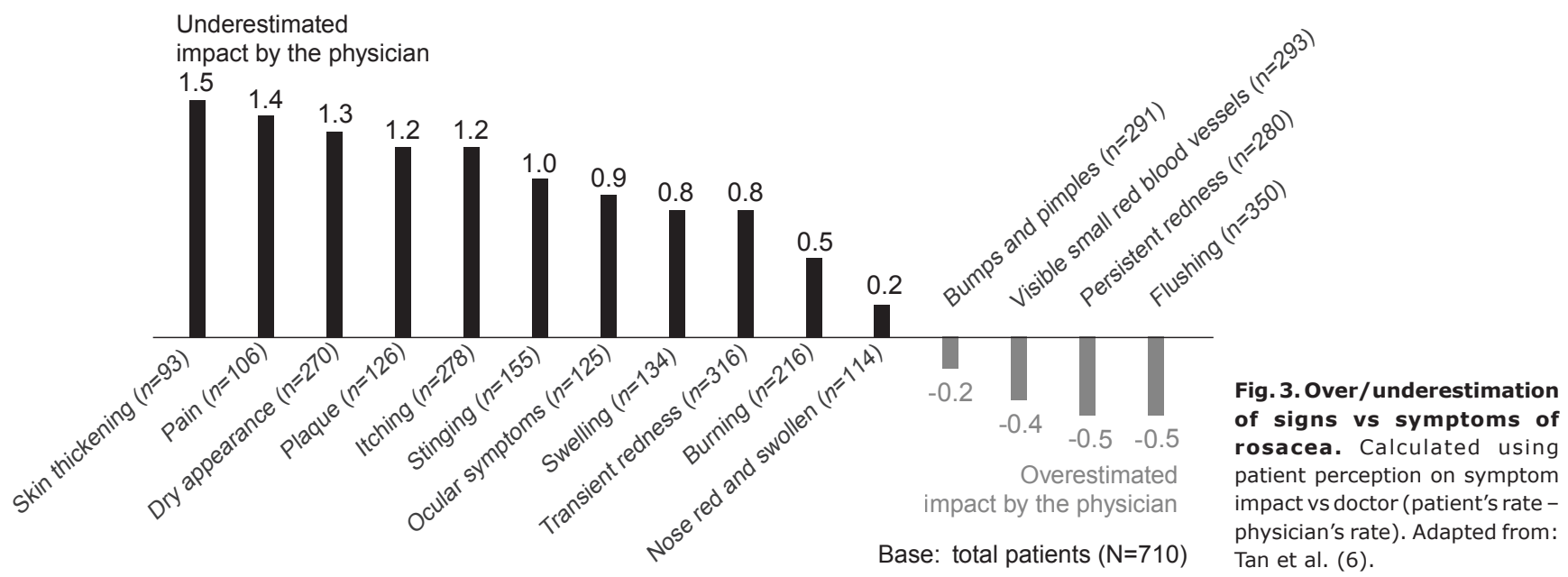


Table IV. 2019 ROSCO phenotype-based treatment approach (Adapted from: Schaller et al. (1) Schaller et al. (45))

\begin{tabular}{|c|c|c|c|c|c|c|c|}
\hline \multirow{2}{*}{$\begin{array}{l}\text { Erythema } \\
\text { Topical }\end{array}$} & & \multicolumn{3}{|c|}{ Inflammatory papules/pustules } & \multirow[t]{2}{*}{ Telangiectasia } & \multicolumn{2}{|l|}{ Phyma } \\
\hline & & & & & & & \\
\hline Transient $^{a}$ & Persistent ${ }^{\mathrm{b}}$ & Mild & Moderate & Severe & Mild, moderate \& severe & Clinically inflamed & Clinically non-inflamed \\
\hline \multirow[t]{2}{*}{ Alpha-adrenergics ${ }^{a}$} & $\begin{array}{l}\text { Alpha-adrenergics: } \\
\text { brimonidine }\end{array}$ & Ivermectin & Ivermectin & Ivermectin & & & \\
\hline & $\begin{array}{l}\text { Alpha-adrenergics: } \\
\text { oxymetazoline (48) }\end{array}$ & $\begin{array}{l}\text { Azelaic acid } \\
\text { Metronidazole }\end{array}$ & $\begin{array}{l}\text { Azelaic acid } \\
\text { Metronidazole }\end{array}$ & & & & \\
\hline \multicolumn{8}{|l|}{ Oral } \\
\hline \multirow[t]{2}{*}{ Beta-blockers $^{a}$} & & Doxycycline ${ }^{\mathrm{c}}$ & Doxycycline $e^{c}$ & Doxycycline $e^{c}$ & & Doxycyclinec & \\
\hline & & & & Isotretinoin & & Isotretinoin & \\
\hline \multirow[t]{2}{*}{ Other } & & & & & & & \\
\hline & $\begin{array}{l}\text { IPL }^{d} \\
P D L\end{array}$ & & & & $\begin{array}{l}\text { IPL }^{\mathrm{d}} \\
\text { Electrodessication } \\
\text { Lasers }^{\mathrm{d}, \mathrm{e}}\end{array}$ & & Physical modalities \\
\hline
\end{tabular}

In a pooled analysis of 1,366 subjects with rosacea from 4 randomized controlled trials, data at the end of treatment (no particular rosacea treatment) showed that more "clear" (IGA 0) than "almost clear" (IGA 1) subjects had a clinically meaningful difference in their Dermatology Life Quality Index (DLQI) score (59\% vs $44 \% ; p<0.001$ ). The DLQI score ranges from 30 (extremely large impact on subject QoL) to 0 (no impact). A change in DLQI score of at least 4 points is considered the minimal clinically important difference. Furthermore, $18 \%$ more "clear" subjects than "almost clear" subjects reported no effect on QoL $(p<0.001)$ (49).

The 2019 ROSCO consensus recommendations also highlighted the importance of achieving "clear" (IGA 0) vs "almost clear" (IGA 1) with a recommendation to set IGA 0 as the primary treatment aim in rosacea in order to minimize disease impact on patients' QoL, maximize time to disease relapse and optimize patient satisfaction with treatment (1). The extent to which achieving "clear" (IGA 0) directly impacts burning and stinging experienced by patients needs to be explored in future studies in order to better understand the therapeutic benefits of treatment on reducing the symptoms of rosacea.

\section{AVAILABLE TREATMENTS CAN INDUCE BURNING AND/OR STINGING}

Many prescription treatments available for rosacea can cause burning and/or stinging, further increasing the burden on patients with rosacea $(50,51-62)$. In a recent global survey exploring patients with rosacea with at least a moderate disease burden $(n=300), 42 \%$ reported that itching and/or burning was a reason for non-compliance with their prescribed treatment. As such, clinicians should consider the impact of each treatment option on the occurrence of these events when deciding the treatment approach (2). Burning and/or stinging are reported as adverse reactions in the summary of product characteristics (SmPCs)/prescribing information (PI) for common rosacea treatments (Table V) $(51-53,55-59,62)$.

\section{Azelaic acid}

Reports of burning and/or stinging exist in the published literature for patients treated with azelaic acid $15 \%$ gel $(54,63,64)$. This has been well defined as a neurosensory property that can affect some patients. In the vehiclecontrolled, phase III azelaic acid $15 \%$ gel studies in

Table V. Rosacea treatments and burning and /or stinging-related adverse effect

\section{Erythematous rosacea}

Brimonidine tartrate $3 \mathrm{mg} / \mathrm{g}$ gel (51)

Oxymetazoline hydrochloride $1 \%$ cream (48)

Papulopustular rosacea

Minocycline $1.5 \%$ foam (indicated for inflammatory lesions) (52)

Azelaic acid $15 \%$ gel $(53,54)$

Azelaic acid $20 \%$ cream (licensed for the topical treatment of acne) (55)

Azelaic acid $15 \%$ foam (56)

Ivermectin $10 \mathrm{mg} / \mathrm{g}$ cream $(57,58)$

Metronidazole $0.75 \%$ gel (59)

Doxycycline $40 \mathrm{mg}$ hard capsules (60)

Doxycycline $100 \mathrm{mg}$ dispersible tablets (61) (licensed for the treatment of acne)

Isotretinoin $10 \mathrm{mg}$ soft capsules (licensed for the treatment of acne) (62)
Application site erythema, pruritus, flushing and skin burning sensation are common adverse reactions, reported in $1.2-3.3 \%$ of patients in clinical studies

No mention of stinging or burning in the PI

Burning/stinging - mild: $13.3 \%$ of patients, moderate: $2.8 \%$ of patients, none reporting severe burning and stinging

Application site burning - very common $(\geq 1 / 10)$

Application site burning - very common $(\geq 1 / 10)$

Application site pain (burning, stinging, paraesthesia and tenderness) in $6.2 \%$ of patients

Skin burning sensation - common $(\geq 1 / 100$ to $<1 / 10)$

Irritation will subside after 1 week

Burning, pain of skin/stinging - common $(\geq 1 / 100$ to $<1 / 10)$

No mention of stinging or burning in SmPCs

Skin irritation (pruritus, rash, dermatitis, dry skin, skin fragility, skin exfoliation) which may have associated symptoms - very common $(\geq 1 / 10)$

PI: prescribing information; SmPCs: summary of product characteristics. 
patients with moderate PPR $(\mathrm{N}=333)$, the most common treatment-related, cutaneous adverse events (incidence $\geq 10 \%$ ) included burning and stinging. Burning, stinging or itching were experienced by $38 \%$ (statistical significance not available) of patients in the azelaic acid group, an incidence higher than in the vehicle-controlled group. In $70 \%$ of this subset of patients, these adverse events were transient in nature and mild to moderate in intensity. Furthermore, $0.6 \%$ of patients reported persistent and severe burning and stinging. Skin care was not controlled in these pivotal studies with azelaic acid 15\% gel (54). The use of moisturizers is recommended to reduce the severity and duration of burning and stinging in patients treated with azelaic acid 15\% gel (63).

Patients with rosacea being treated with azelaic acid foam ( $\mathrm{N}=54,74.1 \%$ of whom were PPR patients), a newer formulation of this topical treatment, completed an online survey of 3 questionnaires including the Rosacea Treatment Preference Questionnaire (RTPQ), Treatment Satisfaction with Medicines Questionnaire (SATMED-Q), and Dermatology Life Quality Index (DLQI). Patientreported side-effects still included stinging $(7.4 \%)$ or burning $(3.7 \%)$, with mean importance scores of 2.5 and 7.0 , respectively (on a 10 -point scale, with 0 meaning no importance and 10 meaning most important) (65).

\section{Metronidazole or azelaic acid}

In patients with rosacea who had at least one prescription for topical metronidazole gel/cream or azelaic acid gel (60.7\% of whom were patients with PPR) who completed a one-time online survey, the most common treatmentrelated concerns included burning sensations $(8.8 \%$ of total responders). A total of $9.2 \%, 6.8 \%$ and $11.1 \%$ of patients receiving metronidazole gel, metronidazole cream and azelaic acid gel were concerned about burning sensations, respectively. Stinging sensations were less of a concern (3.2\% of total responders), with $6.2 \%$ of patients in the metronidazole gel group expressing concern and no patients in the other 2 groups. Using the RTPQ across the study cohort to assess treatment-related concerns, burning received a mean score of 7.4/10, comparable with soreness $(7.6 / 10)$, itching $(7.5 / 10)$ and dryness (7.3/10) (66).

\section{Ivermectin}

In the 2 pivotal, vehicle-controlled, 12-week, phase III ivermectin $10 \mathrm{mg} / \mathrm{g}$ cream studies in moderate-to-severe PPR, treatment-emergent burning related to the study drug was reported less frequently in the ivermectin vs vehicle group $(67,68)$. The most common treatment-related adverse event in study 1 was skin burning, although the frequency of occurrence was lower than the vehicle group (1.8\% vs $2.6 \%$ ) (67). In study 2 , skin burning was reported in $0.2 \%$ of the ivermectin group vs $1.7 \%$ of the vehicle group (68).
In a 40-week, investigator-blinded extension of the phase III studies, treatment-emergent burning related to the study drug was lower in the ivermectin $10 \mathrm{mg} / \mathrm{g}$ cream vs vehicle/azelaic acid $15 \%$ gel group. Over the 40 -week extension in study 1 , burning was reported in $0.2 \%$ of the ivermectin group vs $0.5 \%$ of the azelaic acid gel group, and in study 2 was reported in $0.5 \%$ of the ivermectin group vs $1.4 \%$ of the azelaic acid gel group (69). It is important to note that this data cannot be directly compared as the data refer to 52 weeks of continuous ivermectin $10 \mathrm{mg} / \mathrm{g}$ cream therapy vs 40 weeks of continuous azelaic acid $15 \%$ gel therapy after 12 weeks of pre-treatment on vehicle. However, the overall reduction in burning with ivermectin over the long-term might provide further treatment benefits (67-69).

\section{CHANGE IN BURNING AND/OR STINGING OVER TIME}

In clinical studies of PPR treatment, published data on burning and/or stinging in subjects at both baseline and as outcomes at follow-up visits to address potential improvement of these symptoms over time are more limited.

\section{Metronidazole}

In a phase IV, open-label, multicentre, community-based study of subjects with mild to moderately severe PPR treated with topical metronidazole gel, stinging was evaluated at baseline and week 12 using question 1 in the DLQI. Subjects reported a $25 \%$ improvement in itching, pain, soreness or stinging overall from baseline to week 12 (70).

\section{Ivermectin}

In the phase III, investigator-blinded study comparing the efficacy and safety of ivermectin cream $10 \mathrm{mg} / \mathrm{g}$ once-daily with metronidazole $7.5 \mathrm{mg} / \mathrm{g}$ cream twicedaily ( $\mathrm{N}=962)$ (ATTRACT study), the incidence of worsening from baseline to week 16 for burning/stinging was higher in the metronidazole group vs the ivermectin group (15.5\% vs $11.1 \%)$ (71). When including a 36-week extension, the incidence of worsening from baseline was still lower for ivermectin vs metronidazole $(10.6 \%$ vs $13.6 \%)(72)$.

Previously unpublished data from the ivermectin $10 \mathrm{mg} / \mathrm{g}$ cream phase III studies and their 40 -week extension demonstrated that the proportion of subjects reporting no burning/stinging increased from baseline to week 12 in both groups (Fig. 4). Study 1 demonstrated a greater improvement in the ivermectin vs vehicle group. Study 2 suggesting a more comparable increase (68).

In the ivermectin group, a further increase from week 12 to week 52 in the proportion of subjects reporting no burning/stinging was observed in both studies; an increase in the vehicle/azelaic acid group was observed 

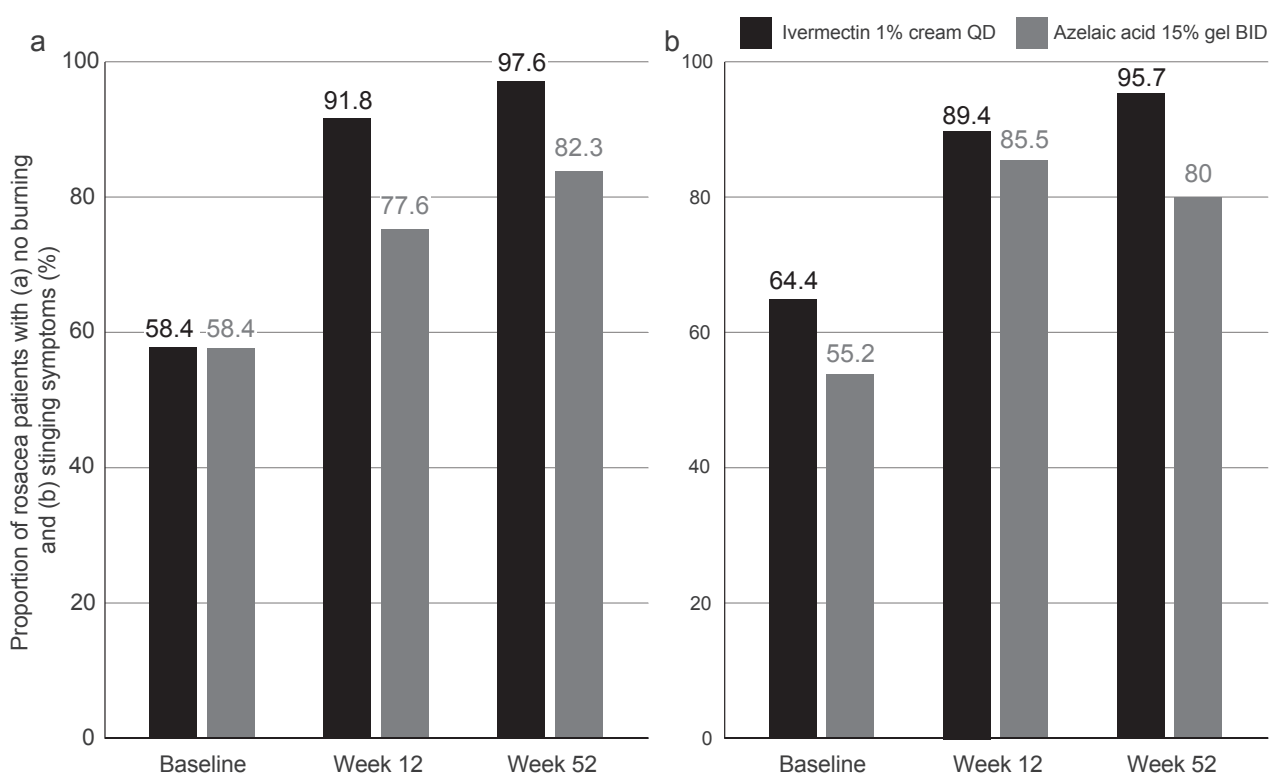

Fig. 4. Proportion of patients with rosacea with no burning/stinging symptoms in the ivermectin 10 $\mathrm{mg} / \mathrm{g}$ cream once a day (QD) group vs azelaic acid $15 \%$ gel twice a day (BID) group; (a) study 1 ; (b) study 2 (68). in only one study (Fig. 4). Treatments cannot be directly compared with one another when assessing reduction in burning/stinging, as subjects in the azelaic acid $15 \%$ gel group were initially treated with vehicle cream for 12 weeks (68).

At week 12, more subjects treated with vehicle vs ivermectin had burning/stinging scores worse than baseline; $\sim 9 \%$ vs $\sim 4 \%$, respectively, in study 1 and $\sim 6 \%$ vs $\sim 4 \%$, respectively, in study 2 . In both studies, the percentage of subjects with burning/stinging scores worse than baseline at each study visit across the 40 -week extension was higher with vehicle/azelaic acid vs ivermectin, which persisted to week 52 (68).

The ANSWER phase IIIb/IV study evaluated ivermectin $10 \mathrm{mg} / \mathrm{g}$ cream as monotherapy vs combination therapy with doxycycline $40 \mathrm{mg}$ modified-release capsules (sub-antibiotic dose doxycycline) in severe rosacea subjects (IGA 4) $(\mathrm{N}=273)$ and systematically included burning/stinging as a secondary endpoint. Ivermectin significantly decreased burning/stinging sensations alone, or in combination with sub-antibiotic dose doxycycline, in most subjects. At baseline, more than $85 \%$ of subjects reported burning/stinging. Most subjects experienced a reduction in burning/stinging from baseline, with approximately $74 \%$ of subjects in both groups being symptomfree at week 12 ( $p<0.001$ for both treatments; post-hoc analysis). At baseline, subjects experienced approximately 5 episodes of flushing per week and the proportion of subjects without flushing increased from baseline to week 12 by $47.2 \%$ with combination therapy and by $41.9 \%$ with monotherapy ( $p<0.001$ for both treatments; post-hoc analysis) (4). The 2019 ROSCO consensus recommendations include burning as a symptom that can co-exist with flushing/transient erythema (1).

The ANSWER post-hoc analysis established a significant positive correlation between burning/stinging sensations and the total DLQI score, as well as individual parameters of the DLQI (Fig. 5). Significant correlations between the reduction in burning/stinging and individual DLQI parameters were found for itchy/painful skin, problems with partner/friends, feeling embarrassed, and social activities. Significant correlations between "clear" (IGA 0) or "almost clear" (IGA 1) and individual DLQI parameters were found for feeling embarrassed $(-0.253$; $p=0.000)$, interference with shopping/home and garden $(-0.161 ; p=0.012)$, and social activities $(-0.143$; $p=0.026)(49)$.

These findings are indirectly confirmed by the results of the ANSWER study, which included subjects with papules and pustules (inflammatory lesions). As expected, there was an improvement in inflammatory lesions, but interestingly also of erythema, flushing, burning and stinging in both treatment arms although ivermectin or ivermectin in combination with doxycycline are primarily used for the treatment of inflammatory lesions (4).

These data demonstrate that continued treatment can alleviate burning and stinging over time, highlighting the need for further exploration of the impact of different PPR treatments on these symptoms in a clinical trial setting. This should be highlighted to the patient when addressing rosacea treatment to emphasize the importance of adherence in managing both the signs and symptoms of rosacea.

\section{CONCLUSION}

Although increasing evidence is available to highlight the negative effect of burning and stinging on patients' QoL, clinicians often do not consciously consider their impact and are not consistently including them as part of their rosacea assessment and disease management approach $(6,49)$. The 2019 ROSCO panel highlighted 


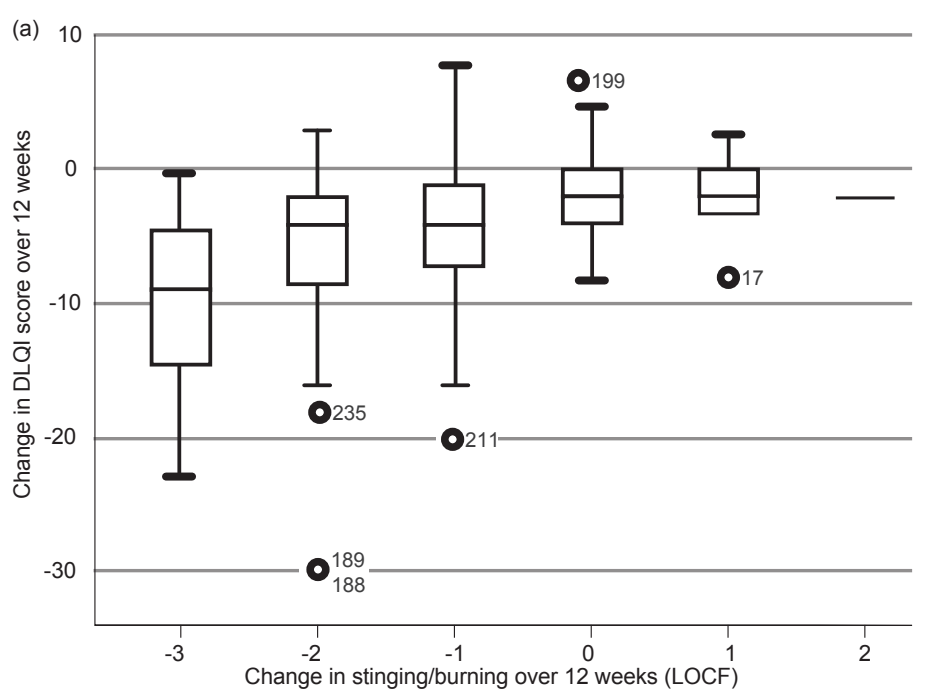

(b)

(b)
\begin{tabular}{|l|l|l|}
\hline \multirow{2}{*}{$\begin{array}{l}\text { Change in stinging/burning } \\
\text { over 12 weeks (LOCF) }\end{array}$} & $\begin{array}{l}\text { Change in DLQI score over } \\
12 \text { weeks }\end{array}$ \\
\cline { 2 - 3 } & P-value & 0.325 \\
\cline { 2 - 3 } & $\mathrm{N}$ & 0.000 \\
\hline
\end{tabular}

(c)

\begin{tabular}{|c|c|c|}
\hline \multirow{4}{*}{$\begin{array}{l}\text { Change inhow itchy, sore, } \\
\text { painful, or stinging has your } \\
\text { skin been? }\end{array}$} & & over 12 weeks (LOCF) \\
\hline & Correlation coefficient & 0.338 \\
\hline & $p$-value & 0.000 \\
\hline & $\mathrm{N}$ & 243 \\
\hline \multirow{3}{*}{$\begin{array}{l}\text { Change in: how much has } \\
\text { your skin created problems } \\
\text { with your partner or any } \\
\text { of your close friends or } \\
\text { relatives? }\end{array}$} & Correlationcoefficient & 0.247 \\
\hline & p-value & 0.000 \\
\hline & $\mathrm{N}$ & 243 \\
\hline \multirow{3}{*}{$\begin{array}{l}\text { Change in: how embarrassed } \\
\text { or selfconscious have you } \\
\text { been because of your skin? }\end{array}$} & Correlation coefficient & 0.243 \\
\hline & p-value & 0.000 \\
\hline & $\mathrm{N}$ & 243 \\
\hline \multirow{3}{*}{$\begin{array}{l}\text { Change in: how much has } \\
\text { your skin affected any social } \\
\text { or leisure activities? }\end{array}$} & Correlation coefficient & 0.237 \\
\hline & p-value & 0.000 \\
\hline & $\mathrm{N}$ & 243 \\
\hline
\end{tabular}

Fig. 5. Impact of a reduction in burning/stinging on the Dermatology Life Quality Index (DLQI) score; (a) Change in DLQI score over 12 weeks vs change in stinging/burning score over 12 weeks; (b) Spearman's correlation for the change in DLQI score vs change in stinging/burning score over 12 weeks; (c) Spearman's correlation between individual DLQI parameters and stinging/burning over 12 weeks (the 4 parameters with strongest correlations are shown). Analyses were performed on the combined treatment arms. Adapted from: Del Rosso et al. (49). LOCF: last observation carried forward.

that non-visible rosacea features, such as burning and stinging, need to be considered as part of any severity assessment (1); however, a practical tool to score burning and stinging related to rosacea does not exist.

Recent scientific evidence from clinical studies highlights that some treatment options have the potential to improve burning and stinging symptoms. Most of these data have been studied with topical ivermectin, as discussed above $(4,68,70)$. Furthermore, evidence also supports the importance of managing epidermal barrier dysfunction associated with rosacea with appropriate skincare, which, in turn, can help to relieve burning and stinging symptoms $(34-36,39,40)$.

Further investigation is needed to fill data gaps; improve our understanding of how to reduce the impact of burning, stinging, and other symptoms of rosacea; and to better incorporate consistent assessment of these symptoms so they are included as part of rosacea management. It is believed, based on information available to date, that this approach will improve patient outcomes in rosacea.

\section{ACKNOWLEDGEMENTS}

Manuscript preparation and editorial assistance was provided to the authors by Abira Sittampalam, Lisa Swanson and Rebecca Diez of Havas Life Medicom, UK and was funded by Galderma SA, Switzerland. This review paper was funded by Galderma SA, Switzerland.

Conflicts of interest: MS reports grants and personal fees from Galderma, personal fees from Abbvie, personal fees from Bayer Healthcare, personal fees from Infectopharm, personal fees from Lilly, outside the submitted work. TD reports personal fees from Almirall, personal fees from Biofrontera, personal fees from Galderma, personal fees from Meda, personal fees from Schulze, personal fees from Bohm GmbH, personal fees from GSK, personal fees from Infectopharm, personal fees from Leo, personal fees from Meda, personal fees from Neracare, personal fees from Novartis, personal fees from Janssen-Cilag, personal fees from Riemser, personal fees from Dr. Pfleger, personal fees from Scibase, outside the submitted work. GM reports personal fees from Galderma, outside the submitted work. LS-G reports grants and personal fees from Galderma, grants and personal fees from Sol Gel, grants and personal fees from Vyne, grants and personal fees from Sun, grants and personal fees from Ortho Derm, grants and personal fees from Almirall, grants and personal fees from Novartis, outside the submitted work. JT reports personal fees from Bausch, personal fees from Leo, personal fees from L'oreal, personal fees from Galderma, personal fees from Promius, personal fees from Sun, outside the submitted work. JDR reports other from Aclaris, other from Almirall, other from Amgen (Celgene), other from Anaptys Bio, other from Arcutis, other from Athenex, other from Bausch (Ortho Dermatology), other from Biofrontera, other from Biopharmx, other from Biorasi, other from Botanix, other from Bricknell, other from Cara Therapeutics, other from Cassiopea, other from Encore, other from Epi Health, other from Ferndale, other from Genentech, other from Incyte, other from Leo Pharma, other from La Roche Posay, other from Lilly (Dermira), other from MC2, other from Novan, other from Pfizer, other from Ralexar, other from Regeneron, other from Sanofi-Genzyme, other from SolGel, other from Sonoma (Intraderm), other from Sun Pharma, other from UCB, other from Verrica, other from Vyne (Foamix/Menlo), other from Galderma, outside the submitted work. SL-R has no conflicts of interest to declare.

\section{REFERENCES}

1. Schaller M, Almeida L, Bewley A, Cribier B, Del Rosso J, Dlova $B$, et al. Recommendations for rosacea diagnosis, classification and management: update from the global ROSacea COnsensus 2019 panel. Br J Dermatol 2020; 182: 1269-1276.

2. Steinhoff M, Harper J, Gieler U, Tan J. The BMJ Hosted Content 2020. Beyond the visible: rosacea and psoriasis of the face [accessed March 2021]. Available from: https://hosted.bmj. com/rosaceabeyondthevisible.

3. Jansen T, Plewig G. Rosacea: classification and treatment. J R Soc Med 1997; 90: 144-150.

4. Schaller M, Kemény L, Havlickova B, Jackson J, Ambroziak 
$M$, Lynde $C$, et al. A randomized phase $3 b / 4$ study to evaluate concomitant use of topical ivermectin $1 \%$ cream and doxycycline $40-\mathrm{mg}$ modified-release capsules, versus topical ivermectin $1 \%$ cream and placebo in the treatment of severe rosacea. J Am Acad Dermatol 2020; 82: 336-343.

5. Tan J, Steinhoff M, Bewley A, Gieler U, Rives V. Characterizing high-burden rosacea subjects: a multivariate risk factor analysis from a global survey. J Dermatolog Treat 2020; 31: 168-174.

6. Tan J, Steinhoff M, Bewley A, Gieler U. The BMJ Hosted Content 2018. Rosacea: beyond the visible [accessed March 2021]. Available from: https://hosted.bmj.com/rosaceabeyondthevisible.

7. Del Rosso J, Tanghetti E, Webster G, Stein Gold L, Thiboutot $D$, Gallo R. Update on the management of rosacea from the American Acne \& Rosacea Society (AARS). J Clin Aesthet Dermatol 2019; 12: 17-24.

8. Wilkin J, Dahl M, Detmar M, Drake L, Feinstein A, Odom R, et al. Standard classification of rosacea: report of the national rosacea society expert committee on the classification and staging of rosacea. J Am Acad Dermatol 2002; 46: 584-587.

9. Del Rosso J, Tanghetti E, Baldwin H, Rosrigues D, Ferrusi I. The burden of illness of erythematotelangiectatic rosacea and papulopustular rosacea: findings from a web-based survey. J Clin Aesthet Dermatol 2017; 10: 17-31.

10. Scharschmidt T, Yost J, Truong S, Steinhoff M, Wang K, Berger T. Neurogenic Rosacea: a distinct clinical subtype requiring a modified approach to treatment. Arch Dermatol 2011; 147: 123-126.

11. Del Rosso J, Thiboutot D, Gallo R, Webster G, Tanghetti E, Eichenfield $L$, et al. Consensus recommendations from the American Acne \& Rosacea Society on the management of rosacea, Part 1: A status report on the disease state, general measures, and adjunctive skin care. Cutis 2013; 92: 234-240.

12. Tan J, Almeida L, Bewley A, Cribier B, Dlova N, Gallo R, et al. Updating the diagnosis, classification and assessment of rosacea: recommendations from the global ROSacea COnsensus (ROSCO) panel. Br J Dermatol 2017; 176: 431-438.

13. Lonne-Rahm S, Fischer T, Berg M. Stinging and rosacea. Acta Derm Venereol 1999; 79: 460-461.

14. Del Rosso J, Gallo R, Kircik L, Thiboutot D, Baldwin H, Cohen $\mathrm{D}$. Why is rosacea considered to be an inflammatory disorder? The primary role, clinical relevance, and therapeutic correlations of abnormal innate immune response in rosacea-prone skin. J Drugs Dermatol 2012; 11: 694-700.

15. Rainer B, Kang S, Chien A. Rosacea: Epidemiology, pathogenesis, and treatment. Dermatoendocrinol 2017; 9: e1361574.

16. Steinhoff $M$, Buddenkotte J, Aubert J, Sulk $M$, Novak $P$, Schwab V, et al. Clinical, cellular, and molecular aspects in the pathophysiology of rosacea. J Investig Dermatol Symp Proc 2011; 15: 2-11.

17. Buddenkotte J, Steinhoff $M$. Recent advances in understanding and managing rosacea. F1000Res 2018; 7: 1885.

18. Forton F, Seys B. Density of Demodex folliculorum in rosacea: a case-control study using standardized skin-surface biopsy. $\mathrm{Br}$ J Dermatol 1993; 128: 650-659.

19. Bonnar E, Eustace P, Powell F. The Demodex mite population in rosacea. J Am Acad Dermatol 1993; 28: 443-448.

20. Forton F, Germaux M, Brasseur T, De Liever A, Laporte M, Mathys $C$, et al. Demodicosis and rosacea: epidemiology and significance in daily dermatological practice. J Am Acad Dermatol 2005; 52: 74-87.

21. Forton F. Papulopustular rosacea, skin immunity and Demodex: pityriasis folliculorum as a missing link. J Eur Acad Dermatol Venereol 2012; 26: 19-28.

22. Casas C, Paul C, Lahfa M, Livideanu B, Lejeune O, AlvarezGeorges S, et al. Quantification of Demodex folliculorum by PCR in rosacea and its relationship to skin innate immune activation. Exp Dermatol 2012; 21: 906-910.

23. Lacey N, Russell-Hallinan A, Zouboulis C, Powell F. Demodex mites modulate sebocyte immune reaction: possible role in the pathogenesis of rosacea. $\mathrm{Br}$ J Dermatol 2018; 179:
420-430.

24. Schaller M, Gonser L, Belge K, Braunsdorf C, Nordin R, Scheu $A$, et al. Dual anti-inflammatory and anti-parasitic action of topical ivermectin 1\% in papulopustular rosacea. J Eur Acad Dermatol Venereol 2017; 31: 1907-1911.

25. Del Rosso J. Topical ivermectin: Data supporting dual modes of action in rosacea. J Clin Aesthet Dermatol 2017; 10: 39-42.

26. Koller B, Müller-Wiefel A, Rupec R, Korting H, Ruzicka T. Chitin modulates innate immune responses of keratinocytes. PLoS One 2011; 6: e16594.

27. Forton F, De Maertelaer V. Erythematotelangiectatic rosacea may be associated with a subclinical stage of demodicosis: a case-control study. $\mathrm{Br}$ J Dematol 2019; 181: 818-825.

28. Cardwell L, Alinia H, Tuchayi S, Feldman S. New developments in the treatment of rosacea - role of once-daily ivermectin cream. Clin Cosmet Investig Dermatol 2016; 9: 71-77.

29. Berardesca E, Farage M, Maibach H. Sensitive skin: an overview. Int J Cosmet Sci 2013; 35: 2-8.

30. Van Zuuren E. Rosacea. N Engl J Med 2017; 377: 1754-1764.

31. Schaller M, Pietschke K. Successful therapy of ocular rosacea with topical ivermectin. $\mathrm{Br}$ J Dermatol 2018; 179: 520-521.

32. Addor F. Skin barrier in rosacea. An Bras Dermatol 2016; 91: 59-63.

33. Zhou M, Xie $H$, Cheng $L$, Li J. Clinical characteristics and epidermal barrier function of papulopustular rosacea: A comparison study with acne vulgaris. Pak J Med Sci 2016; 32: $1344-1348$.

34. Del Rosso J, Levin J. The clinical relevance of maintaining the functional integrity of the stratum corneum in both healthy and disease-affected skin. J Clin Aesthet Dermatol 2011; 4: 22-42.

35. Dirschka T, Tronnier H, Fölster-Holst R. Epithelial barrier function and atopic diathesis in rosacea and perioral dermatitis. $\mathrm{Br}$ J Dermatol 2004; 150: 1136-1141.

36. Levin J, Miller R. A guide to the ingredients and potential benefits of over-the-counter cleansers and moisturizers for rosacea patients. J Clin Aesthet Dermatol 2011; 4: 31-49.

37. Picardo M, Eichenfield L, Tan J. Acne and rosacea. Dermatol Ther (Heidelb) 2017; 7: 43-52.

38. Yuan C, Ma Y, Wang Y, Qian C, Hocquet D, Zheng S, et al. Rosacea is associated with conjoined interactions between physical barrier of the skin and microorganisms: a pilot study. J Clin Lab Anal 2020; 34: e23363.

39. Wollina U. Recent advances in the understanding and management of rosacea. F1000Prime Rep 2014; 6: 50.

40. Baldwin H, Santoro F, Lachmann N, Teissedre S. A novel moisturizer with high sun protection factor improves cutaneous barrier function and the visible appearance of rosaceaprone skin. Cosmet Dermatol 2019; 18: 1686-1692.

41. Tan J, Berg M, Gallo R, Del Rosso J. Applying the phenotype approach for rosacea to practice and research. $\mathrm{Br}$ J Dermatol 2018; 179: 741-746.

42. Gallo R, Granstein R, Kang S, Mannis M, Steinhoff M, Tan J, et al. Standard classification and pathophysiology of rosacea: the 2017 update by the National Rosacea Society Expert Committee. J Am Acad Dermatol 2018; 78: 148-155.

43. Sannino $M$, Lodi $G$, Dethlefsen $M$, Nisticò $S$, Cannarozzo G, Nielsen M. Fluorescent light energy: treating rosacea subtypes 1, 2, and 3. Clin Case Rep 2018; 6: 2385-2390.

44. Chen H, Lai W, Zheng Y. Rosacea in acne vulgaris patients: subtype distribution and triggers assessment - a crosssectional study. J Cosmet Dermatol 2021; 20: 1889-1896.

45. Schaller M, Almeida L, Bewley A, Cribier B, Dlova N, Kautz G, et al. Rosacea treatment update: recommendations from the global ROSacea COnsensus (ROSCO) panel. Br J Dermatol 2017; 176: 465-471.

46. Del Rosso J, Thiboutot D, Gallo R, Webster G, Tanghetti E, Eichenfield $L$, et al. Consensus recommendations from the American acne \& rosacea society on the management of rosacea, Part 2: a status report on topical agents. Cutis 2013; 92: 277-284

47. Asai Y, Tan J, Baibergenova A, Barankin B, Cochrane C, 
Humphrey S, et al. Canadian clinical practice guidelines for rosacea. J Cutan Med Surg 2016; 20: 432-445.

48. Del Rosso J, Jackson M, Johnson S, Harvey A, Chavda R. Effect of combination therapy on visible/non-visible symptoms, and disease burden associated with severe rosacea: results from a post-hoc analysis of a randomized controlled trial. SKIN J Cutan Med 2020; 4: s56.

49. Webster G, Schaller M, Tan J, Jackson J, Kerrouche N, Schäfer G. Defining treatment success in rosacea as "clear" may provide multiple patient benefits: results of a pooled analysis. J Dermatolog Treat 2017; 28: 469-474.

50. Allergan. RHOFADE $®$ (oxymetazoline hydrochloride) $1 \%$ Cream Prescribing Information [accessed 2021 Mar]. Available from: https://www.accessdata.fda.gov/drugsatfda_docs/ label/2017/208552s000lbl.pdf.

51. Galderma. MIRVASO ${ }^{\circledR}$ (brimonidine tartrate) $3 \mathrm{mg} / \mathrm{g} \mathrm{Gel}$ Summary of Product Characteristics [accessed 2021 Mar]. Available from: https://www.medicines.org.uk/emc/product/5303/smpc.

52. Foamix. ZILXI ${ }^{\circledR}$ (minocycline) $1.5 \%$ Foam Full Prescribing Information [accessed 2021 Mar]. Available from: https://www.accessdata.fda.gov/drugsatfda_docs/ label/2020/213690s000lbl.pdf.

53. Leo Laboratories Ltd. FINACEA $\AA$ (azelaic acid) $15 \%$ Gel Summary of Product Characteristics [accessed 2021 Mar]. Available from: https://www.medicines.org.uk/emc/product/240/smpc.

54. Thiboutot D, Thieroff-Ekerdt R, Graupe K. Efficacy and safety of azelaic acid $(15 \%)$ gel as a new treatment for papulopustular rosacea: results from two vehicle-controlled, randomized phase III studies. J Am Acad Dermatol 2003; 48: 836-845.

55. Leo Laboratories Ltd. SKINOREN ${ }^{\circledR}$ (azelaic acid) 20\% Cream Summary of Product Characteristics [accessed 2021 Mar]. Available from: https://www.medicines.org.uk/emc/product/285/smpc\# : : text=Topical\%20treatment $\% 20$ of\%20 acne $\% 20$ vulgaris.\&text=Skinoren $\% 20$ Cream $\% 20$ should $\% 20$ be $\% 20$ applied,for\%20the $\% 20$ entire $\% 20$ facial\%20area.

56. Leo Pharma Inc. FINACEA $®$ (azelaic acid) 15\% Foam Prescribing Information [accessed 2021 Mar]. Available from: https://www.accessdata.fda.gov/drugsatfda_docs/ label/2015/207071s000lbl.pdf.

57. Galderma. SOOLANTRA $®$ (ivermectin) $10 \mathrm{mg} / \mathrm{g}$ Cream Summary of Product Characteristics [accessed $2021 \mathrm{Mar}$. Available from: https://www.medicines.org.uk/emc/medicine/30353.

58. Galderma. SOOLANTRA $®$ Ivermectin $10 \mathrm{mg} / \mathrm{g}$ Cream Australia Prescribing Information [accessed 2021 Mar]. Available from: https://www.galderma.com/au/sites/g/files/ jcdfhc266/files/inline-files/Soolantra\%20PI.pdf.

59. ADVANZ Pharma. ZYOMET® (metronidazole) $0.75 \% \mathrm{w} / \mathrm{w}$ Gel Summary of Product Characteristics [accessed 2021 Mar]. Available from: https://www.medicines.org.uk/emc/ product/512/smpc.
60. Galderma. EFRACEA ${ }^{\circledR}$ (doxycycline monohydrate) $40 \mathrm{mg}$ Modified release hard capsules summary of product characteristics [accessed 2021 Mar]. Available from: https://www. medicines.org.uk/emc/medicine/22268.

61. Pfizer Ltd. VIBRAMYCIN-D $®$ (doxycycline monohydrate) 100 mg Hard Capsules Summary of Product Characteristics [accessed 2021 Mar]. Available from: https://www.medicines. org.uk/emc/product/4063\#gref.

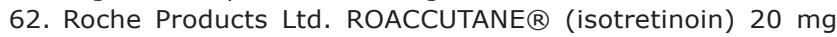
Soft Capsules Summary of Product Characteristics [accessed $2021 \mathrm{Mar}$. Available from: https://www.medicines.org.uk/ emc/product/6470/smpc.

63. Del Rosso J. The use of moisturizers as an integral component of topical therapy for rosacea: clinical results based on the assessment of skin characteristics study. Cutis 2009; 84: 72-76.

64. Draelos Z. Noxious sensory perceptions in patients with mild to moderate rosacea treated with azelaic acid $15 \%$ gel. Cutis 2004; 74: 257-260

65. Williamson T, Cameron J, McLeod K, Turner B, Quillen A, LaRose $A$, et al. Concerns and treatment satisfaction in patients being treated with azelaic acid foam for rosacea. J Drugs Dermatol 2019; 18: 381-386.

66. Williamson T, Cheng W, McCormick N, Vekeman F. Patient preferences and therapeutic satisfaction with topical agents for rosacea: a survey-based study. Am Health Drug Benefits 2018; 11: 97-106.

67. Stein Gold L, Kircik L, Fowler J, Tan J, Draelos Z, Fleischer A et al. Efficacy and safety of ivermectin $1 \%$ cream in treatment of papulopustular rosacea: results of two randomized, double-blind, vehicle-controlled pivotal studies. J Drugs Dermatol 2014; 13: 316-323.

68. Galderma R\&D, LCC. RD.06.SRE.18170 and RD.06. SRE.18171. Data on file.

69. Stein Gold L, Kircik L, Fowler J, Jackson J, Tan J, Draelos Z, et al. Long-term safety of ivermectin $1 \%$ cream vs azelaic acid $15 \%$ gel in treating inflammatory lesions of rosacea: results of two 40-week controlled, investigator-blinded trials. J Drugs Dermatol 2014; 13: 1380-1386.

70. Wolf JE, Del Rosso JQ. The CLEAR trial: results of a large community-based study of metronidazole gel in rosacea. Cutis 2007; 79: 73-80.

71. Taieb A, Ortonne JP, Ruzicka T, Roszkiewicz J, Berth-Jones J, Peirone $M$, et al. Superiority of ivermectin $1 \%$ cream over metronidazole $0.75 \%$ cream in treating inflammatory lesions of rosacea: a randomized, investigator-blinded trial. $\mathrm{Br}$ J Dermatol 2015; 172: 1103-1110.

72. Taieb A, Khemis A, Ruzicka T, Barańska-Rybak W, Berth-Jones J, Schauber J, et al. Maintenance of remission following successful treatment of papulopustular rosacea with ivermectin $1 \%$ cream vs. metronidazole $0.75 \%$ cream: 36 -week extension of the ATTRACT randomized study. J Eur Acad Dermatol Venereol 2016; 30: 829-836. 